\title{
Visualizando el desarrollo de la nanomedicina en México
}

\author{
Eduardo Robles-Belmont', Rebeca de Gortari-Rabiela², Pilar Galarza-Barrios', \\ Jesús Mario Siqueiros-García y Alejandro Arnulfo Ruiz-León ${ }^{1}$ \\ ${ }^{1}$ Instituto de Investigaciones en Matemáticas Aplicadas y en Sistemas, Laboratorio de Redes, ${ }^{2}$ Instituto de Investigaciones Sociales, Universidad
} Nacional Autónoma de México. Ciudad de México, México

\section{Resumen}

Objetivo: En este artículo efectuamos diversas visualizaciones de datos sobre la producción científica en el campo de la nanomedicina en México. Método: Las visualizaciones presentadas se basan en diversas metodologías para el análisis y la visualización de datos, tales como el análisis de redes sociales, los mapas geográficos de la ciencia y los análisis de comunidades. Resultados: Los resultados muestran un panorama del desarrollo de la nanomedicina en México desde diversas entradas y visualizaciones. Además, las visualizaciones expuestas en los resultados identifican tendencias y patrones en las colaboraciones nacionales e internacionales, en la estructura del conocimiento científico a partir de las áreas temáticas y disciplinas presentes, así como en la identificación de comunidades científicas que desarrollan nuevos conocimientos en este campo emergente en México.

PALABRAS CLAVE: Nanomedicina. Visualización de datos. Tecnologías emergentes. México.

\section{Abstract}

Objective: In this article we present a set of different visualizations of Mexico's nanomedicine scientific production data. Method: Visualizations were developed using different methodologies for data analysis and visualization such as social network analysis, geography of science maps, and complex network communities analysis. Results: Results are a multi-dimensional overview of the evolution of nanomedicine in Mexico. Moreover, visualizations allowed to identify trends and patterns of collaboration at the national and international level. Trends are also found in the knowledge structure of themes and disciplines. Finally, we identified the scientific communities in Mexico that are responsible for the new knowledge production in this emergent field of science.

KEY WORDS: Nanomedicine. Data visualization. Emerging technologies. Mexico.

\section{Introducción}

A lo largo de la progresión de las nuevas ciencias y tecnologías (СуT), el discurso que promueve su avance se basa principalmente en las potenciales aplicaciones. En el caso de la emergencia de las nanociencias y nanotecnologías (NCT), el discurso se ha centrado en las potencialidades de estas en términos técnicos, económicos y sociales en prácticamente todos los sectores. Las aplicaciones propuestas de las nanotecnologías se fundamentan en las nuevas propiedades físicas, químicas y eléctricas de los materiales a escala nanométrica (mil millonésima parte de un metro, 10-9). Las nanotecnologías son consideradas como una ventana de oportunidad para impulsar el desarrollo industrial y responder a ciertas necesidades sociales y medioambientales. Esto ha llamado la atención de diversos actores provenientes de instituciones gubernamentales, de la industria y de otros actores sociales. La promoción de las nanotecnologías ha sido sustentada en algunos países a través de iniciativas nacionales para el financiamiento y la orientación de las investigaciones científicas y tecnológicas hacia sectores considerados como estratégicos.

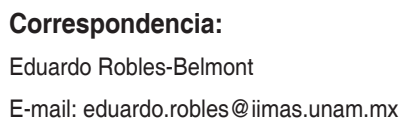

Fecha de recepción en versión modificada: 20-12-2016

Fecha de aceptación: 30-12-2016

DOI://dx.doi.org/10.24875/GMM.17002851
Gac Med Mex. 2017;153:875-885

Contents available at PubMed www.gacetamedicademexico.com 
En el sector salud, las nanotecnologías han encontrado diversas aplicaciones que van desde los fármacos hasta las prótesis y los dispositivos para diagnóstico. En los medios de divulgación científica se han cubierto las aplicaciones en el diagnóstico y las terapias para el cáncer y otras enfermedades consideradas como problemas de salud pública. En México, el desarrollo de las NCT se inició en la década de 1990 y han participado las instituciones del sector salud $^{1}$. Además, otros estudios han mostrado que diversos grupos de investigación desarrollan nuevos conocimientos en las diferentes subáreas de la nanomedicina $^{2}$, y las redes científicas en este campo reflejan colaboraciones con los países que han encabezado el desarrollo de estas nuevas tecnologías ${ }^{3}$.

Por otro lado, frente a la emergencia de las nuevas CyT es necesario tener indicadores sobre su progresión y tendencias, tanto para el apoyo a las políticas en CyT como para el estudio de estas desde las ciencias sociales. El tema de los indicadores sobre CyT emergentes no es nuevo. Existen diversos grupos de investigación que desarrollan propuestas metodológicas para el mapeo de la emergencia, de la interdisciplinariedad, de las capacidades disponibles, de la discusión académica, de las relaciones de colaboración científica y de aspectos e implicaciones sociales.

La bibliometría y la cienciometría son dos disciplinas dedicadas al estudio y la valoración de la producción científica. Al medir la diversidad de publicaciones se han generado indicadores y enfoques teóricos sobre el funcionamiento de la СуT. Sobre los aportes de estas disciplinas, existe una amplia literatura académica ${ }^{4,5}$. La producción de indicadores también ha sido tarea de agencias gubernamentales e internacionales, cuyos objetivos son la política y la gestión del desarrollo científico y tecnológico. El interés por los indicadores de estos actores institucionales se ha centrado en la formulación de recomendaciones para la política en CyT ${ }^{6-8}$. Además, los indicadores son usados para la evaluación de la productividad y el desempeño de las actividades académicas de instituciones, grupos e investigadores. Estrechamente relacionado con los usos antes mencionados, los indicadores también se utilizan en estudios de prospectiva y de evaluación de tecnologías emergentes con el fin de identificar capacidades y necesidades para abordar ventanas de oportunidad en la emergencia de nuevas tecnologías.

En este contexto, el avance en las ciencias de la información y de la computación ha significado aportes importantes. En particular, el desarrollo del
Internet y de la web, así como de la web social, ha puesto al alcance de la investigación grandes cantidades de información y datos que son relevantes para el estudio de la CyT. El ámbito académico y tecnológico ha generado sus propias herramientas en el contexto de la web social; si bien servicios como Twitter son ampliamente utilizados para difundir, compartir y comentar una multiplicidad de temas vinculados con la ciencia, existen otros medios que han sido generados específicamente para la CyT. Algunos ejemplos de ello son Mendeley, Github o Figshare. El primero está centrado en compartir y manejar material bibliográfico, especialmente artículos; el segundo es un sistema de versionado, repositorio y plataforma colaborativa para el desarrollo de software de todo tipo, pero que es ampliamente utilizado para cómputo científico; y el tercero es un repositorio de acceso a datos generados en la investigación. El potencial que representa el acceso a la información que se genera en la web social solo tiene sentido porque actualmente se cuenta con el poder computacional y las herramientas para su análisis y visualización. Por último, la apuesta con estas tecnologías es que se puedan desarrollar indicadores que informen sobre los procesos y las dinámicas de la generación y la socialización del conocimiento en el lapso de horas y días, de manera masiva. En otras palabras, a diferencia de las escalas espaciales y temporales de la cienciometría y la bibliometría, estas tecnologías permiten observar el transcurso de la ciencia a una escala espacial muy amplia y temporal muy corta.

La representación gráfica de la información para su visualización es una actividad común en la mayoría de las disciplinas científicas. Sin embargo, el uso de las representaciones gráficas en combinación con la tecnología informática para conseguir una adecuada visualización de la información es relativamente nuevo. Los avances en las ciencias de la información y de la computación ya mencionados han desembocado en el desarrollo de herramientas computacionales visuales que incluyen hardware, software, herramientas de interfaces visuales para gráficos y técnicas, así como el procesamiento de datos. Resultado del planteamiento del gran volumen de datos con que se contaba (dilema de la información sin interpretación) desde mediados de los años 1980, a iniciativa de la National Science Foundation de los EE.UU. se propuso desarrollar dicha tecnología, lo que además implicó la colección, la organización, la modelación y la representación ${ }^{9}$. A partir de ello ha habido grandes avances, ya que ha cobrado importancia en diferentes disciplinas para la 
comprensión de los procesos y sus relaciones complejas. Las formas en las que se puede medir y representar la visibilidad científica se han transformado de los indicadores ordenados en tablas y gráficos, que contienen información, ideas, etc., a la representación mediante mapas de redes, donde la información puede convertirse en una base de conocimiento. La visualización de redes sociales puede ser una importante herramienta para la explicación de los datos, ya que ayuda al investigador a tamizarlos, detectar patrones de interés y dar sentido a las observaciones ${ }^{10}$. Además, permite la comprensión de estructuras completas de datos y de las relaciones entre los diferentes elementos, así como la formulación de hipótesis que pueden convertirse en objeto de análisis ${ }^{11}$. El análisis de datos relacionales sobre la producción científica puede aportar conocimiento que permite complementar el análisis bibliométrico y cientométrico con el análisis de redes sociales y otros análisis de datos. Frente a estas nuevas herramientas para el análisis de datos emerge la pregunta sobre cuál es su aporte para la producción de indicadores de CyT en campos emergentes. Otras cuestiones que nos interesan son cuáles son las áreas de especialización y su papel en la colaboración, qué instituciones están detrás y qué papel desempeñan en las redes las políticas de fomento y colaboración, por mencionar algunas.

Partiendo de estas dos bases, la emergencia de la nanomedicina y la visualización de datos para nuevos indicadores de CyT, en este artículo presentamos los resultados de un estudio sobre la producción de nuevos conocimientos científicos y tecnológicos en el campo de la nanomedicina en México hasta el año 2014. En otros trabajos ya se ha analizado la emergencia y el desarrollo de la nanomedicina en México ${ }^{2,3,12}$, cuyos resultados aportan un panorama amplio de este campo emergente en el país. En este estudio, los resultados se presentan bajo la forma de diversas visualizaciones de diferentes datos bibliométricos sobre las áreas de la nanomedicina y sus relaciones, la dimensión espacial de las colaboraciones científicas en esta tecnología emergente, las colaboraciones institucionales, las disciplinas presentes y el mapeo de comunidades científicas. Las visualizaciones expuestas en este trabajo complementan otros estudios efectuados sobre el desarrollo de la nanomedicina en México.

\section{Metodos}

Este estudio se basa en el uso de herramientas bibliométricas y cienciométricas para analizar datos sobre las publicaciones científicas publicadas en el campo de la nanomedicina, producidos en México e indizados en Web of Science. La identificación de las referencias bibliográficas se ha efectuado a partir de la estrategia de búsqueda propuesta por Wagner, et al. ${ }^{13}$, la cual ha sido replicada para el estudio de la emergencia de la nanomedicina en América Latina y el Caribe $^{2}$, y emplea diversas palabras clave que son representativas de siete áreas concernientes a la salud humana: administración de fármacos; fármacos y terapia; imagenología in vivo; biosensores y biomateriales; implantes inteligentes/prótesis neuronales; y se agregó a la estrategia de búsqueda el término «Nanomedicina» con el objetivo de identificar los trabajos académicos que abordan el tema desde una perspectiva general y desde otras disciplinas de las ciencias sociales. Así mismo, se eliminó el área de cosméticos, ya que no la consideramos un área en el sector salud con implicaciones importantes en sus necesidades.

Por otro lado, recordemos que el objetivo de este estudio es exponer indicadores a través de visualizaciones de datos. Se trata de evitar el uso de tablas e histogramas para exponer los indicadores. Las visualizaciones que presentamos parten del análisis de redes sociales. Estas herramientas nos ayudan a generar visualizaciones de los datos basadas en el análisis relacional de estos. Los resultados presentados son multinivel, ya que exponemos: a) las colaboraciones internacionales, b) las redes de colaboración institucional, y c) las colaboraciones científicas de los diversos investigadores. Además, las visualizaciones reflejan las áreas o disciplinas del conocimiento donde han sido publicados los nuevos conocimientos producidos en el campo de la nanomedicina en México. En su conjunto, estos resultados son conocidos como mapas de la ciencia $^{14,15}$, y visualizarlos desde la perspectiva de las disciplinas presentes en el desarrollo de nuevos conocimientos nos puede revelar las relaciones entre las diversas disciplinas en la producción y la acumulación de nuevos conocimientos. Además, este tipo de visualizaciones pueden aportarnos indicios de las dinámicas en los procesos de convergencia de conocimientos en campos emergentes.

Las visualizaciones se hicieron a partir de diversos programas informáticos y metodologías que permiten obtener mapas geográficos, así como imágenes de análisis de redes sociales y de conglomerados (clusters) y su comparación a lo largo del tiempo. Los análisis de redes sociales han sido efectuados principalmente con el programa Pajek. El mapa 
geográfico de las colaboraciones internacionales se ha basado en la metodología propuesta por Leydesdorff y Persson ${ }^{16}$. Los análisis y la visualización de comunidades han sido efectuados con la metodología de Map Equation ${ }^{17}$. Las referencias de estas herramientas y metodologías serán explicitadas en los apartados de los resultados.

\section{Resultados}

\section{Desarrollo de las áreas de la nanomedicina en México}

Hasta el año 2014, en México se han producido 265 artículos en el campo de nanomedicina. Los primeros artículos fueron publicados a finales de la década de 1990. Sin embargo, es a partir del año 2006 cuando la producción rebasa los 10 artículos anuales y el crecimiento de la producción de artículos se ha incrementado de forma importante a partir del año 2010*. Esto muestra que el campo de la nanomedicina en México se encuentra aún en etapas tempranas de su emergencia.

La primera visualización es sobre la distribución de estos artículos en cinco de las seis áreas de la nanomedicina mapeadas, además del área denominada nanomedicina. En la figura 1 se muestra una red donde cada nodo representa las seis áreas identificadas; su tamaño está determinado en función del número de artículos en cada área y las líneas representan las relaciones entre las áreas, las cuales están basadas en los artículos distribuidos en más de un área. Esta red ha sido generada con Pajek y visualizada con VOSviewer ${ }^{18}$. En esta visualización apreciamos que el área más desarrollada concierne a la administración de fármacos, con prácticamente el $50 \%$ de los artículos. De hecho, esta área es una de las más desarrolladas en la nanomedicina en todo el mundo, cuyas investigaciones científicas están orientadas al desarrollo de la administración dirigida de fármacos, la reducción del efecto tóxico, la seguridad y la biocompatibilidad, así como al desarrollo rápido de medicamentos seguros ${ }^{19}$.

Imagenología en vivo es la segunda área en la nanomedicina en México, la cual representa el 28.3\% de la producción científica. La imagenología ha encontrado un auge con la nanotecnología en el desarrollo de diversas investigaciones en torno al diagnóstico y las terapias para el cáncer. En efecto, diversos proyectos se han centrado en las nuevas propiedades de nanopartículas (p. ej., propiedades magnéticas, electrónicas y ópticas) para diferentes aplicaciones en imagenología en vivo ${ }^{20}$.

Las dos siguientes áreas son las de biomateriales (10.57\%) y biosensores (8.30\%). Los biomateriales han despertado un interés creciente en aplicaciones en la medicina al ser combinados con las nuevas propiedades de los nanomateriales; se trata del desarrollo de sustitutos de tejidos, de biosensores y de sistemas de diagnósticos, así como del suministro controlado de fármacos ${ }^{21}$. Las investigaciones en esta área se han centrado igualmente en atender diversos obstáculos encontrados en el uso de los nanomateriales ${ }^{22}$. El área de menor producción es la de fármacos y terapia (2.64\%), resultado que llama nuestra atención, ya que es en el sector de la farmacéutica donde han surgido las principales promesas de avance con la Nanomedicina, y para el caso de México se trata de una de las dos áreas menos desarrolladas.

También la figura 1 muestra las relaciones entre las áreas identificadas en el campo de la nanomedicina a partir del enfoque propuesto por Wagner, et al. ${ }^{13}$. Este mapa de la nanomedicina nos muestra que todas las áreas están relacionadas con la administración de fármacos, la cual es el área más desarrollada en México. Es decir, se identifican trabajos de investigación realizados y en desarrollo que son transversales al área de administración de fármacos y cada una de las otras áreas identificadas. Este mismo mapa muestra la presencia de investigaciones transversales a las áreas de biomateriales, biosensores y administración de fármacos, lo que refleja que en las líneas de investigación que se desarrollan en México también hay preocupación en torno a los obstáculos en el uso de nanomateriales en medicamentos. Lo anterior podría estar relacionado, por una parte, con la falta de estudios de toxicidad, y por otra parte con la inexistencia de regulaciones y normas.

\section{Colaboraciones científicas}

La segunda visualización que nos interesa revisar concierne a las colaboraciones científicas internacionales. Esta visualización se basa en el análisis de

\footnotetext{
* En este trabajo no presentamos una curva del crecimiento de los artículos producidos en México en el campo de la nanomedicina, ya que el objetivo es explorar nuevas visualizaciones de datos diferentes a las comunes, como son los histogramas. El lector puede consultar otros trabajos en los que se ha analizado la evolución de la producción científica en este campo ${ }^{2,3,12}$.
} 


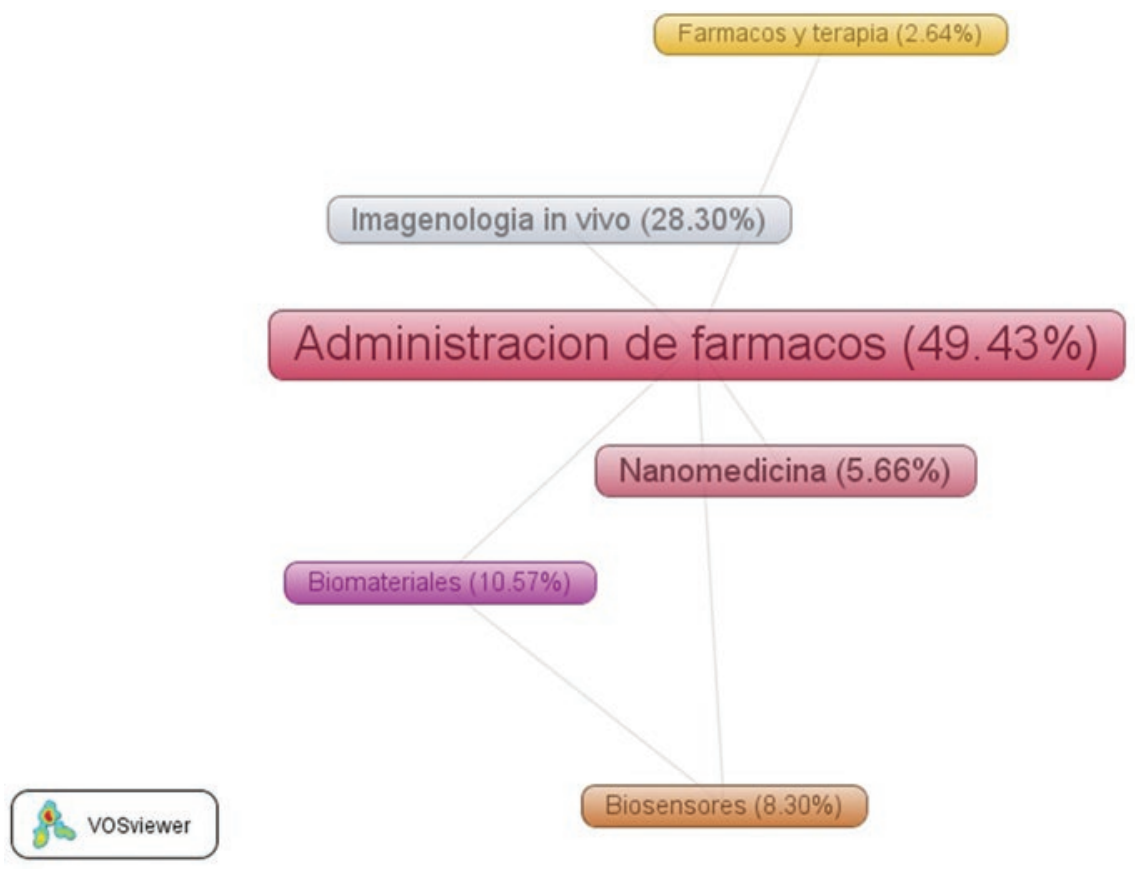

Figura 1. Áreas temáticas de la nanomedicina desarrolladas en México hasta el año 2014 (fuente: Web of Science, Thomson Reuters, febrero 2016; elaboración propia y visualización con VOSviewer).

redes de colaboración, y la metodología parte del trabajo de Leydesdorff y Persson ${ }^{16}$ para efectuar mapas geográficos de la ciencia. En la figura 2 se muestra el mapa geográfico de las colaboraciones internacionales en el desarrollo de la nanomedicina en México. Los datos para construir esta visualización han sido las coautorías de investigadores adscritos a instituciones en México con sus pares adscritos a instituciones extranjeras. Llama nuestra atención las pocas colaboraciones internacionales de los grupos de investigadores mexicanos en este campo. De los 265 artículos publicados hasta el año 2014, solo 61 han sido producidos en colaboración internacional. Aunque hay pocas colaboraciones, las tendencias que presentan estas son similares a las que podemos observar en otros sectores de la nanotecnología o de otras tecnologías emergentes. En esta primera visualización se muestra que las principales colaboraciones se han mantenido con investigadores de los EE.UU. Posteriormente sobresalen las colaboraciones con países europeos. Sin embargo, las colaboraciones con países de la región de América Latina y el Caribe son poco significativas. Dichos resultados pueden deberse a que los investigadores mexicanos se han formado principalmente en los EE.UU. y Europa.

Por otro lado, la visualización de la figura 3 está centrada sobre las colaboraciones institucionales científicas en la nanomedicina en México, tanto nacionales como internacionales. En la parte central de la imagen se encuentran las instituciones educativas y de investigación mexicanas, así como sus relaciones. La Universidad Nacional Autónoma de México (UNAM), el Centro de Investigación y Estudios Avanzados, y el Instituto Politécnico Nacional son las instituciones con mayor presencia en el desarrollo de la nanomedicina en el país. Esto constata una concentración de capacidades y actividades en estas tres entidades mexicanas. A pesar de esta concentración de capacidades, es interesante destacar la distribución de las demás instituciones presentes en el desarrollo de la nanomedicina. Por una parte, los Centros Públicos de Investigación del Consejo Nacional de Ciencia y Tecnología (CONACYT), conformados en su inicio por núcleos de investigadores procedentes de la UNAM principalmente, que han logrado aportar conocimiento en diferentes regiones. Por otra parte, y a partir de los cambios en las políticas de CуТ, las universidades públicas más importantes de diferentes entidades han logrado tener avances importantes en estos campos emergentes. Por último, destaca también la presencia de instituciones de salud y laboratorios federales que participan en este desarrollo, y cuya creación y crecimiento se han basado principalmente en el financiamiento recibido de fuentes gubernamentales.

Por otro lado, la figura 3 revela igualmente una distribución bastante amplia de las colaboraciones científicas internacionales. Esto llama nuestra atención, pues 


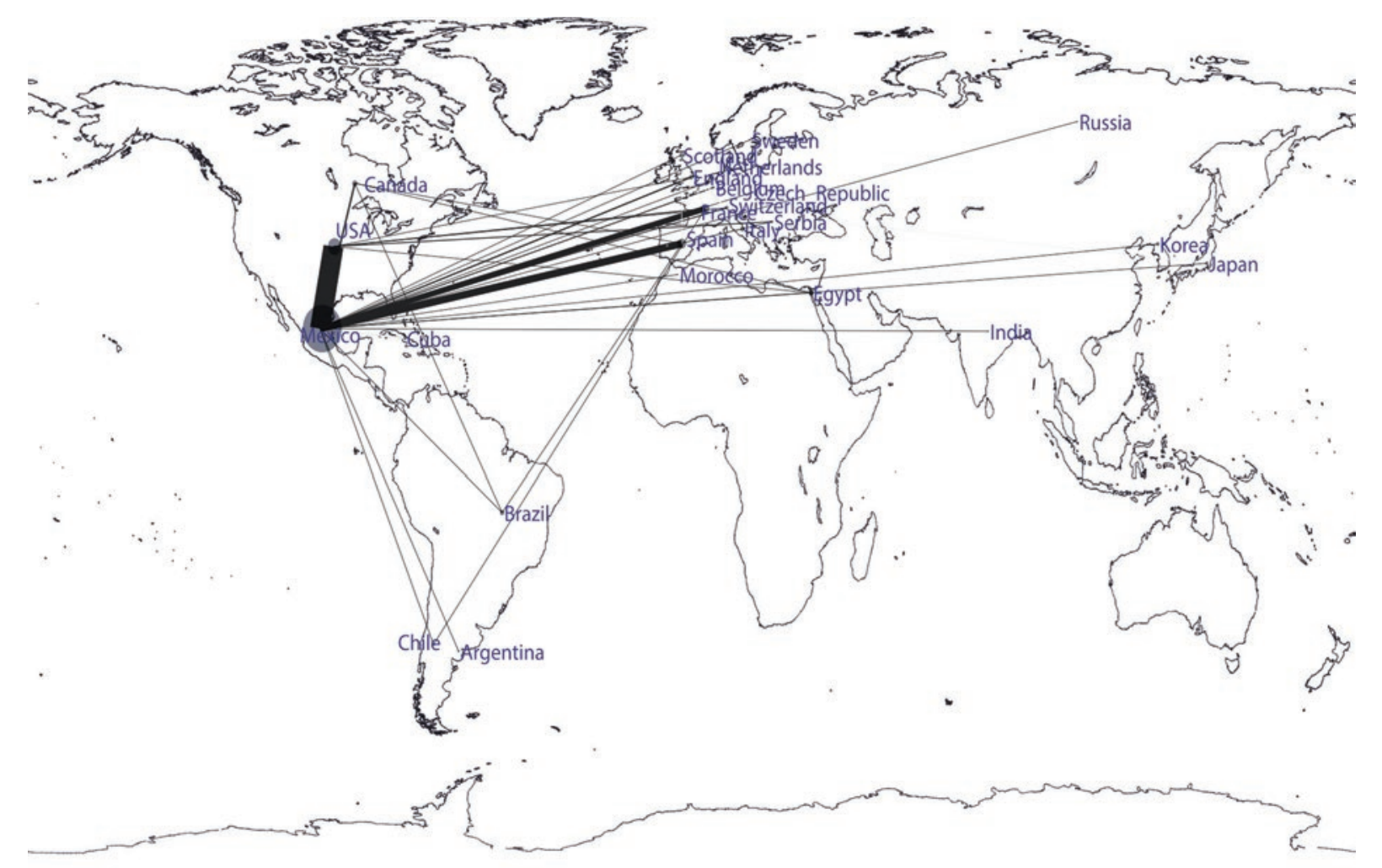

a.

Figura 2. Colaboraciones internacionales en el desarrollo de la nanomedicina en México hasta el año 2014 (fuente: realización propia con datos de Web of Science).

se trata de 61 colaboraciones que son muy recientes, las cuales datan a partir del año 2012 y pueden ser interpretadas como una característica de una reciente internacionalización de la ciencia en la nanomedicina. Al respecto cabe señalar que el desarrollo de las NCT ha sucedido en medio de un contexto de internacionalización y comercialización de la ciencia ${ }^{23-25}$. Además, en estas dinámicas nuevas de la producción, el uso y la diseminación de conocimientos científicos y tecnológicos, en general, se puede hablar de la modificación de las políticas públicas, donde uno de sus pilares es la economía del conocimiento, de tal forma que se busca orientar la ciencia, la tecnología y la innovación hacia la competitividad y los encadenamientos productivos. En el caso de las tecnologías emergentes, como las NCT, a pesar de no existir una iniciativa nacional y con objetivos claros, la investigación en estos campos no solo ha aumentado, sino que se ha diversificado, apoyándose en su multidisciplinariedad ${ }^{26}$. Además, estas redes de colaboración se han beneficiado de la transferencia de conocimientos y capacidades a partir de las relaciones de movilidad con investigadores de otros países, mostrando así que la movilidad internacional permite la formación de redes basadas en el acceso a la infraestructura y las nuevas tecnologías. Finalmente, consideramos que el conjunto de los programas institucionales y nacionales de apoyo al desarrollo de estas tecnologías emergentes, así como la formación de recursos humanos, han contribuido al crecimiento reciente de estas colaboraciones.

\section{Áreas del conocimiento}

Otro indicador interesante que visualizar concierne a las disciplinas y áreas del conocimiento que aportan al desarrollo de СуТ emergentes. En este apartado nos interesa revisar otra perspectiva de la visualización basada en las disciplinas del conocimiento, así como sus relaciones en la producción de nuevos conocimientos. Los resultados de estas técnicas de visualización se conocen como mapas de la ciencia ${ }^{14,15}$, y los datos de entrada son las categorías donde son indizadas las revistas científicas en las cuales han sido publicados los artículos científicos.

En la figura 4 se muestra el mapa de las áreas del conocimiento basado en las categorías de la clasificación de Web of Science. La red de Web of Science ha sido generada con Pajek y analizada con 


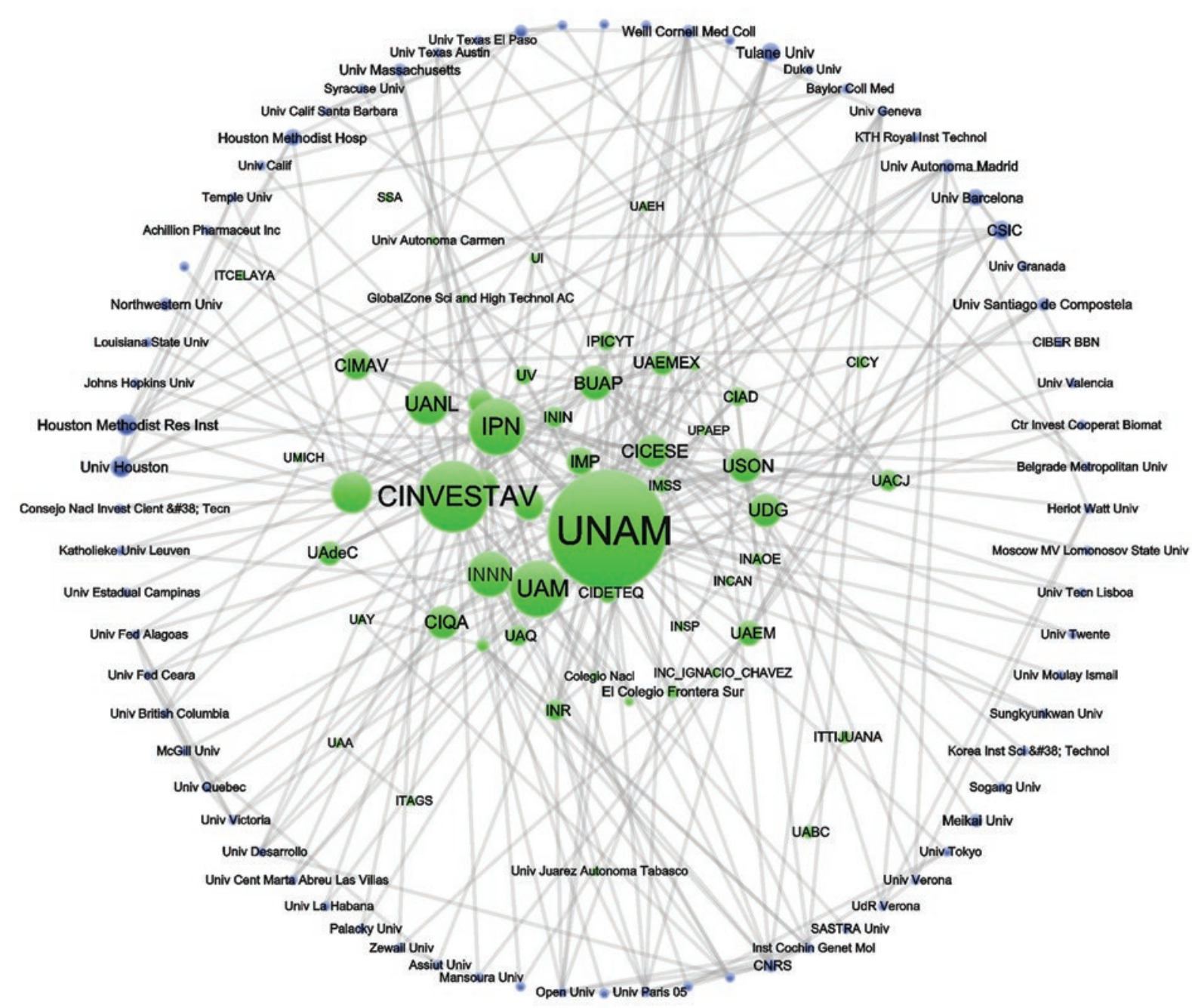

Figura 3. Distribución de las colaboraciones científicas nacionales e internacionales en el campo de la nanomedicina en México hasta el año 2014 (fuente: construcción propia con datos de Web of Science).

el método de identificación de comunidades de Louvain $^{27}$, y la visualización con VOSviewer desarrollado por Waltman y Van Eck ${ }^{18}$. Se identificaron 60 Web of Science en donde están clasificadas las revistas científicas de nuestra muestra (265 artículos), y se aplicó el método de análisis de comunidades de Louvain, basado en la optimización de la modularidad $^{27}$. Este análisis nos arrojó un total de 15 conglomerados (clusters). Estos resultados se muestran en la figura 4, donde el tamaño del nodo está determinado a partir de la frecuencia de las Web of Science, lo que nos indica que la ciencia de materiales es la principal disciplina en el desarrollo de la nanomedicina en México. Otras disciplinas de las ciencias básicas presentes en este mapa conciernen al área de la química, y en menor medida se aprecia la presencia de otras disciplinas como farmacología y farmacia, así como otras disciplinas de las ciencias médicas. Encontramos interesante evocar que la centralidad de la ciencia de los materiales, en el caso mexicano, puede responder a que las NCT se han desarrollado en departamentos, laboratorios y centros con una tradición en esta área. Esto nos lleva a calificarla como el área temática convergente en el desarrollo de la nanomedicina, es decir, aquella con la que más fuentes comparten las demás áreas y la que más conocimiento aporta al resto.

La visualización mostrada en la figura 4 es interesante, ya que nos muestra una imagen de la estructura del conocimiento en el desarrollo de la nanomedicina en México. Sin embargo, un límite que presenta este tipo de visualizaciones es que no permite mostrar la dinámica en el desarrollo de este campo emergente. Se trata, en efecto, de una imagen estática de la estructura del conocimiento en la 


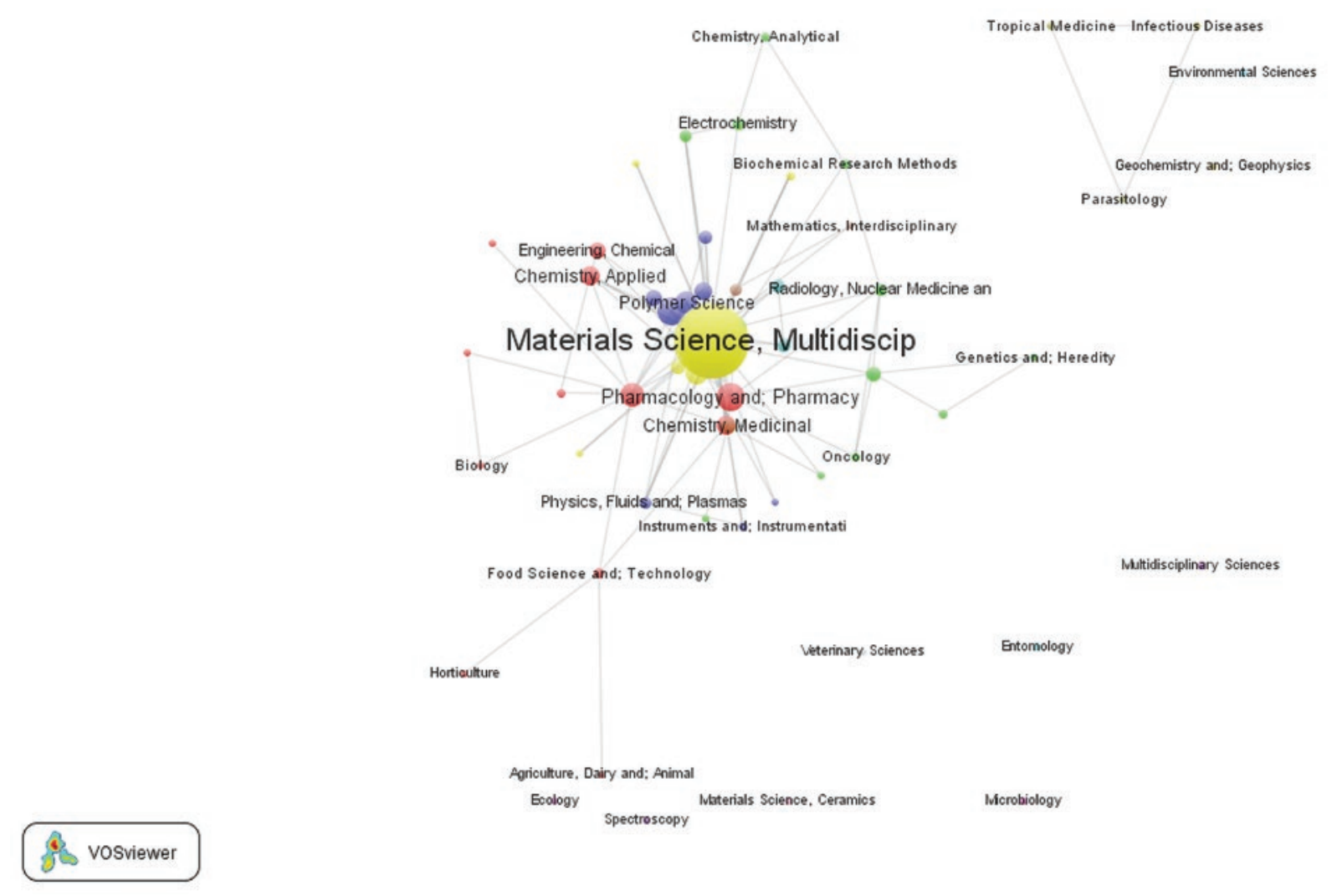

Figura 4. Mapa de las áreas del conocimiento presentes en el desarrollo de la nanomedicina en México hasta el año 2014 (fuente: construcción propia con datos de Web of Science).

nanomedicina. Entonces, con el fin de mostrar una visualización dinámica que nos permita observar el desarrollo de la presencia de disciplinas en el campo de la nanomedicina a través del tiempo en México, hemos usado la herramienta para generar comparaciones en el tiempo de redes sociales propuesta por Rosvall y Bergstrom ${ }^{28}$. Los datos los hemos divido en cuatro periodos, cuyos cortes los hemos efectuado a partir de fechas que consideramos que han marcado el desarrollo de las NCT en México**.

En la figura 5 se muestra el resultado de esta visualización que nos permite observar la dinámica del cambio del perfil del conocimiento de la nanomedicina en México a través del tiempo. Este resultado nos confirma la presencia central de las ciencias de materiales y su evolución en el periodo analizado donde vemos como ha aportando a otras áreas del conocimiento en la nanomedicina. Si complementamos la interpretación de esta visualización con la figura 4, podemos observar que esta área del conocimiento está relacionada con otras áreas de la física, de las ciencias de polímeros y de la química. Por otro lado, los resultados nos muestran que la emergencia de la nanomedicina en el área de los fármacos se ha iniciado a partir de la primera década de este siglo. Además, los conocimientos en otras áreas del conocimiento en esta tecnología emergente han surgido después de esta década.

\section{Comunidades científicas}

La última visualización concierne a las comunidades de investigadores que han participado en el

** El primer corte en el tiempo es el año 2001, que corresponde al lanzamiento de la Iniciativa Nacional para las Nanotecnologías en Ios EE.UU., hecho considerado como el inicio de la carrera internacional por el desarrollo de estas tecnologías emergentes. El segundo corte se ha hecho en el año 2006, en el cual tuvo lugar la instalación de infraestructura importante en el marco de la Iniciativa del COANCYT de Laboratorios Nacionales. El tercer corte se ha hecho en el año 2009, ya que en ese año se crearon las primeras Redes Temáticas del CONACYT, entre las cuales hay al menos dos dedicadas al campo de las nanotecnologías. El último corte lo hemos hecho hasta el año 2014 con el fin de tener datos actualizados y no tener sesgo por la cola de indización, que en el caso de documentos científicos puede reflejarse hasta 2 años después. 


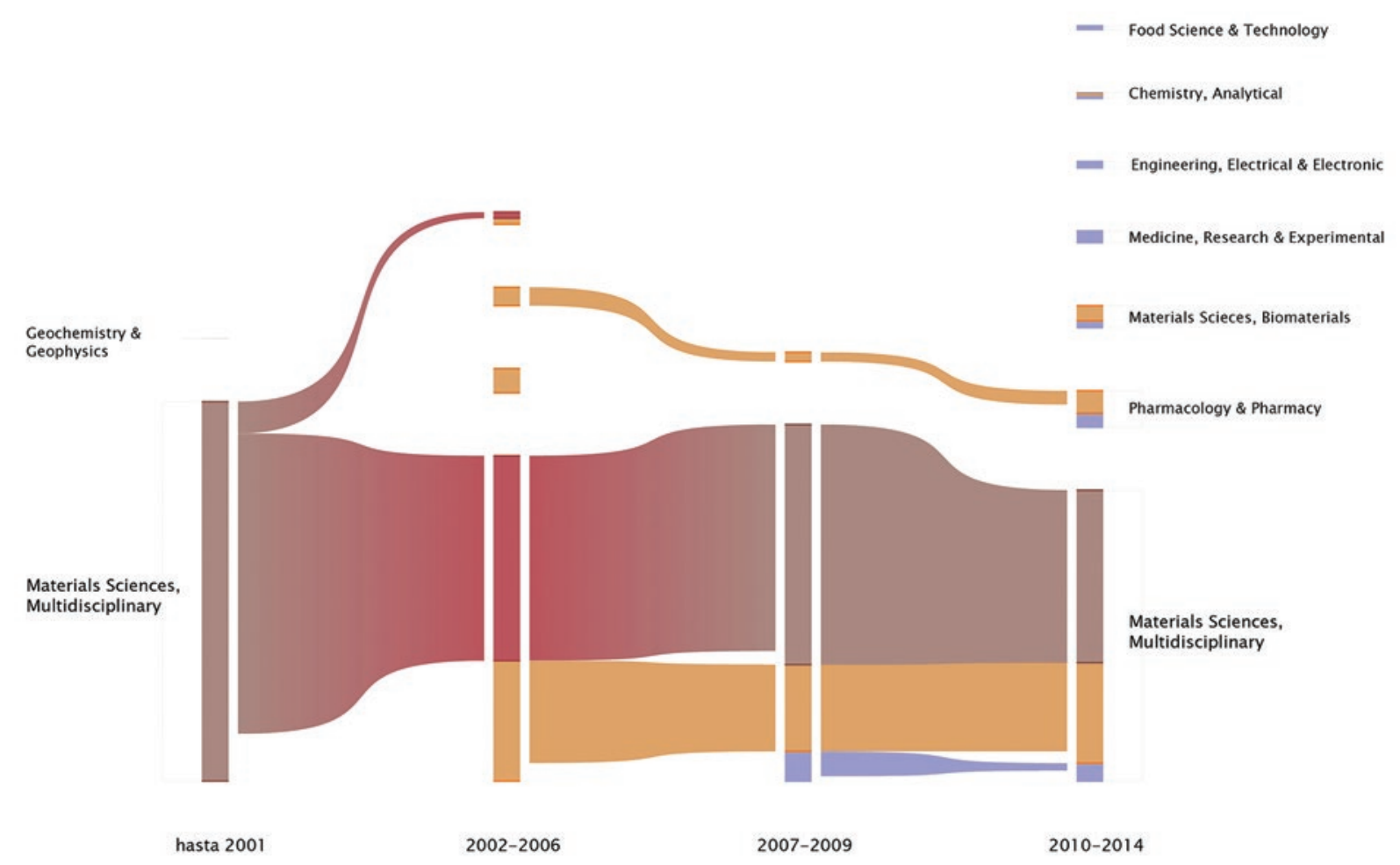

Figura 5. Desarrollo de la estructura del conocimiento en la nanomedicina en México hasta el año 2014 (fuente: construcción propia con datos de Web of Science).

desarrollo del área de la nanomedicina en México. Este ejercicio nos interesa para identificar las diferentes comunidades de investigadores que desarrollan actividades científicas en las diversas áreas de la nanomedicina en México. Esto se realizó a partir de las coautorías de los 265 artículos identificados, con un total de 1050 autores mexicanos y extranjeros.

Este análisis de comunidades ha sido efectuado con el método Map Equation ${ }^{17}$, el cual está basado en el flujo de información en la estructura de una red y cuyas posibles particiones están determinadas por su recorrido al azar. Este análisis nos ha dado como resultado un total de 25 comunidades (Fig. 6). Cada comunidad identificada ha sido etiquetada en el área de la nanomedicina donde han sido caracterizados los artículos publicados. La visualización de estas comunidades nos muestra que la mayoría desarrolla nuevos conocimientos en el área de administración de fármacos (11 comunidades), seguida de imagenología en vivo. Además de identificar las comunidades por área de la nanomedicina, encontramos interesante con esta visualización identificar las relaciones entre las diferentes comunidades. Por ejemplo, en la visualización de la figura 6 podemos apreciar dos grupos heterogéneos conformados por comunidades de diferentes áreas: el primero, cuya principal comunidad es en biomateriales, también tiene relaciones a través de las coautorías de los artículos con las áreas de imagenología en vivo y administración de fármacos; y en el segundo grupo, la principal comunidad trabaja en administración de fármacos y ha mantenido relaciones de colaboración con comunidades en las áreas de biomateriales e imagenología en vivo.

\section{Conclusiones}

En este trabajo hemos constatado que las nuevas herramientas para la visualización de datos son interesantes para los estudios sobre la emergencia y desarrollo de las nuevas CyT. En efecto, su aplicación para el monitoreo y la vigilancia tecnológica, así como para el mapeo en los estudios sociales de la ciencia y la tecnología, permite identificar tendencias, relaciones, fortalezas y debilidades, y los principales actores institucionales y científicos. Además, las visualizaciones de datos con estas nuevas herramientas nos permiten obtener nuevas representaciones de los sistemas científicos y tecnológicos desde diversas entradas y perspectivas, potencializando así las capacidades interpretativas y analíticas de los indicadores de ciencia y tecnología. No obstante, el uso de estas herramientas debe ser tomado con cuidado. Cada 


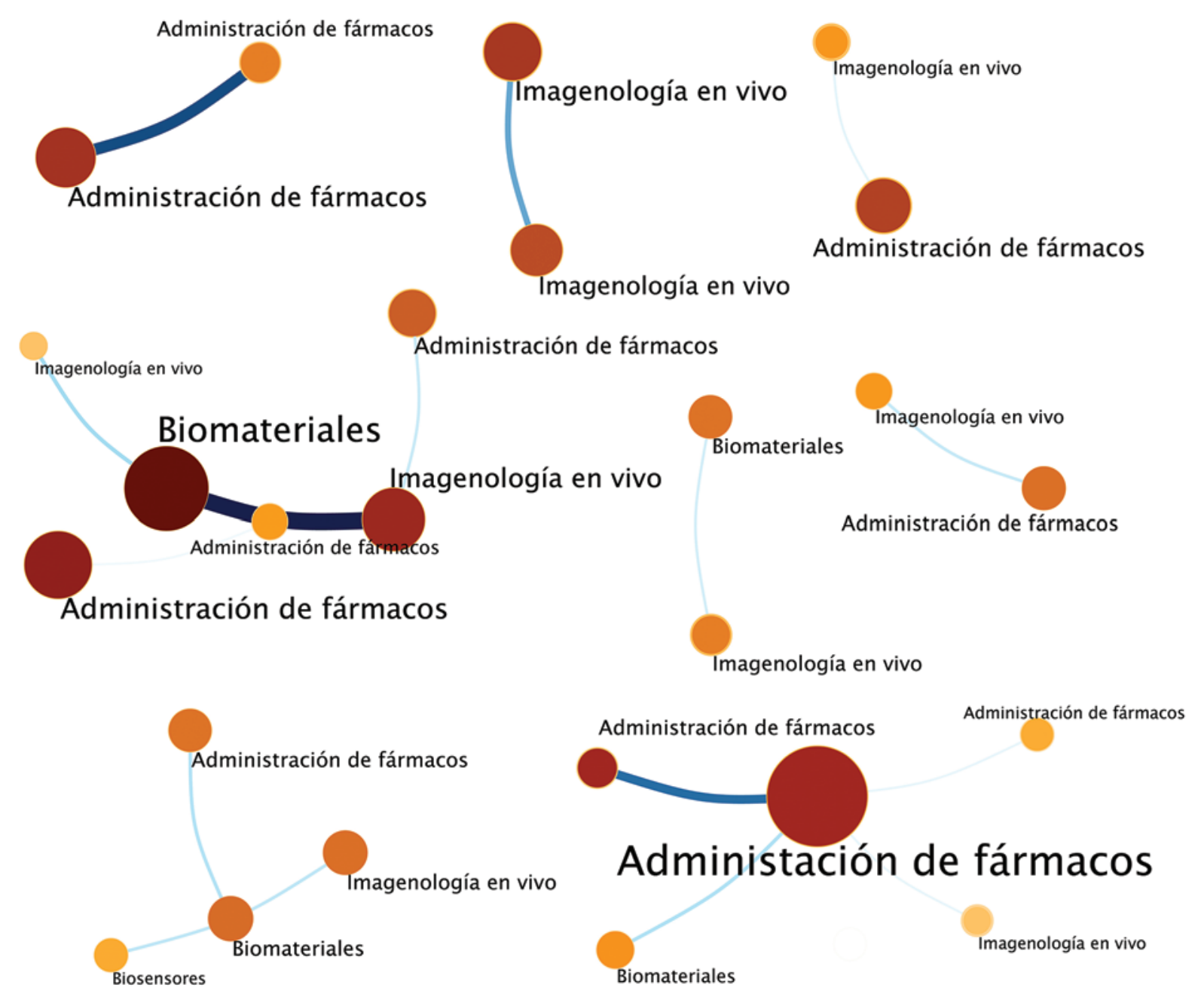

Figura 6. Comunidades científicas en el desarrollo de la nanomedicina en México hasta el año 2014 (fuente: construcción propia con datos de Web of Science).

herramienta tiene características particulares que limitan su uso para diferentes casos. El conocimiento y el uso de diversas herramientas para la visualización de datos permite sobrepasar ciertos límites, lo que hace que las herramientas sean complementarias entre ellas. Cada una de las visualizaciones construidas a partir de diversas entradas nutre la tarea de mapear un campo científico. Por otro lado, cabe mencionar que las metodologías y las herramientas empleadas en este estudio son de acceso libre, lo que abre la posibilidad de reproducir estas visualizaciones en otros estudios.

Concerniente al desarrollo de la nanomedicina en México, las redes de colaboraciones internacionales visualizadas geográficamente nos dan una amplia perspectiva de la distribución geográfica de las relaciones de colaboración científica en el desarrollo de este campo emergente. Los resultados muestran una dinámica reciente de colaboración internacional, la cual indica que las nuevas CyT presentan procesos acelerados de internacionalización. Estos procesos pueden deberse a diferentes factores, como son la visibilidad científica, los acuerdos institucionales de colaboración y el desarrollo de temas en común, entre otros. También es interesante señalar que, al hacer el análisis a diferentes niveles, hemos identificado una distribución institucional amplia y a la vez concentrada en los EE.UU. y algunos países de Europa. Esta configuración de las colaboraciones hace emerger diversas preguntas en torno a las dinámicas del desarrollo de las nuevas CyT y el papel de las relaciones Norte-Sur; tema que ha quedado fuera del alcance de este estudio, pero que es importante señalar.

Además, el análisis de la estructura de este campo emergente desde diversas entradas nos ha mostrado, por un lado, la importancia de las ciencias de 
materiales y de otras disciplinas de la física y la química desde las primeras publicaciones. Llevar este análisis de la estructura de la nanomedicina a otro nivel nos ha mostrado que el desarrollo de fármacos en México ha sido central. Finalmente, el análisis en el tiempo de esta estructura nos ha mostrado una visualización interesante del desarrollo de la nanomedicina y de cómo los nuevos conocimientos han incursionado en diferentes disciplinas científicas. Sin embargo, la ausencia del sector privado en el desarrollo de conocimientos científicos en la nanomedicina en México sugiere que los conocimientos desarrollados son aún básicos.

\section{Agradecimientos}

Esta investigación fue apoyada por el proyecto IA300916 del Programa de Apoyo a Proyectos de Investigación e Innovación Tecnológica (PAPIIT) de la UNAM.

\section{Bibliografía}

1. Robles-Belmont E, Vinck D. A panorama of nanoscience developments in Mexico based on the comparison and crossing of nanoscience monitoring methods. J Nanosci Nanotechnol. 2011;11:5499-507.

2. Invernizzi N, Foladori G, Robles-Belmont E, et al. Nanotechnology for social needs: contributions from Latin American research in the areas of health, energy and water. J Nanoparticle Res. 2015;17:233.

3. Robles-Belmont E. Cooperación científica internacional en la nanomedicina de México. Obs Desarro. 2014:3:29-33.

4. Pritchard A. Statistical bibliography or bibliometrics? J Doc. 1969;25:348-9.

5. Garfield E. Citation indexing: its theory and applications in science, technology and the humanities. New York: John Wiley \& Sons; 1979. 274 p.

6. OECD. Proposed standard practice for surveys on research and experimental development. Frascati Manual. Paris; 2002. Disponible en: http:// www.oecd-ilibrary.org/science-and-technology/frascati-manual-2002_9789264199040-en

7. OECD. Oslo manual. Guidelines for collecting and interpreting innovation data. $3^{\text {rd }}$ ed. Paris: OECD; 2005. 166 p. Disponible en: http://www.oecd-ilibrary.org/science-and-technology/oslo-manual_9789264013100-en

8. Jaramillo $H$, Lugones $G$, Salazar M, editores. Normalización de indicadores de innovación tecnológica en América Latina y el Caribe. Manua de Bogotá. RICYT, OEA, CYTED; 2001. 102 p. Disponible en: http:// www.uis.unesco.org/Library/Documents/Bogota Manual_Spa.pdf

9. Torres Ponpúan D. Aproximaciones a la visualización como disciplina científica. Acimed. 2009;20:161-74.

10. Brandes U, Kenis P. La explicación a través de la visualización de redes. REDES - Revista hispana para el análisis de redes sociales. 2005;9(6). Disponible en: http://revista-redes.rediris.es/html-vol9/vol9_6.htm

11. Moya-Anegón $F$, Vargas-Quesada $B$, Chinchilla-Rodríguez $Z$, et al. Visualización y análisis de la estructura científica española: ISI Web of science 1990-2005. Disponible en: http://www.academia.edu/1430783/ Visualizaci\%C3\%B3n y an\%C3\%A1lisis de la estructura cient\%C3\%ADfica espa\%C3\%B10la ISI Web of Science 1990-2005

12. Robles-Belmont, E. and de Gortari-Rabiela, R. Dynamics of the emergence of micro an nanotechnologies in the healthcare sector in Mexico. Nanotech L \& Bus. 2013; 10:54-64.

13. Wagner V, Husing B, Gaisser S, et al. Nanomedicine: drivers for development and possible impacts. European Commission Joint Research Centre. Sevilla; 2008. Disponible en: http://ftp.jrc.es/EURdoc/JRC46744.pdf

14. Leydesdorff $L$, Rafols I. A global map of science based on the ISI subject categories. J Am Soc Inf Sci Technol. 2009;60:348-62.

15. Bornmann L, Leydesdorff L, Walch-solimena C, et al. Mapping excellence in the geography of science: an approach based on Scopus data. Informetr. 2011;5:537-46.

16. Leydesdorff $L$, Persson $O$. Mapping the geography of science: distribution patterns and networks of relations among cities and institutes. J Am Soc Inf Sci Technol. 2010;61:1622-34.

17. Rosvall M, Axelsson D, Bergstrom CT. The map equation. Eur Phys $J$ Spec Top. 2009;178:13-23.

18. Waltman L, Van Eck NJ. A new methodology for constructing a publication-level classification system of science. J Am Soc Inf Sci Technol. 2012;63:2378-92.

19. De Jong WH, Borm PJ. Drug delivery and nanoparticles: applications and hazards. Int J Nanomedicine. 2008:3:133-49.

20. Cuenca AG, Jiang $H$, Hochwald $S N$, et al. Emerging implications of nanotechnology on cancer diagnostics and therapeutics. Cancer. 2006;107:459-66.

21. Liu H, Webster TJ. Nanomedicine for implants: a review of studies and necessary experimental tools. Biomaterials. 2007;28:354-69.

22. Doshi N, Mitragotri S. Designer biomaterials for nanomedicine. Adv Funct Mater. 2009;19:3843-54.

23. Wong PK, Ho YP, Chan CK. Internationalization and evolution of application areas of an emerging technology: the case of nanotechnology. Scientometrics. 2007;70:715-37.

24. Gómez Uranga M, Etxebarria Kerexeta G, Campàs-Velasco J. The dynamics of commercialization of scientific knowledge in biotechnology and nanotechnology. Eur Plan Stud. 2007;15:1199-214.

25. Fernández-Ribas AA, Shapira $P$. Technological diversity, scientific excellence and the location of inventive activities abroad: the case of nanotechnology. J Technol Transf. 2009;34:286-303.

26. Robles-Belmont E, De Gortari Rabiela R. NST without NII ? The Mexican case study. En: Ramani SV, editor. Nanotechnology: what's in it for emerging countries? Cambridge: Cambridge University Press; 2007. p. 182-210.

27. Blondel VD, Guillaume J-L, Lambiotte R, et al. Fast unfolding of communities in large networks. J Stat Mech Theory Exp. 2008;10008:6. Disponible en: http://arxiv.org/abs/0803.0476

28. Rosvall M, Bergstrom CT. Mapping change in large networks. PLoS One. 2010;5:e8694 